\title{
Solitary necrotic nodules of the liver simulating hepatic metastases
}

\author{
NEIL A SHEPHERD, GARRY LEE \\ From the Department of Histopathology, St Bartholomew's Hospital, West Smithfield, London EC1A 7BE
}

SUMMARY Four cases are described in which solitary nodules were identified on the anterior aspect of the liver. These had characteristic histological appearances. Each had a necrotic core surrounded by a dense collar of hyalinised collagen, incorporating elastic fibres. While three of our patients had carcinoma elsewhere none had evidence of tumour in the liver. We do not believe that these lesions represent hepatic metastases. We suggest that they may be of traumatic aetiology or a sequel of previous infection.

The identification of hepatic metastases at operation may greatly influence the surgeon in his approach to cancer treatment. It would be important to document the existence of a rare but non-tumorous lesion which might easily be misinterpreted as necrotic tumour by the pathologist on frozen (or indeed, paraffin) section.

Over the past four years in our department, we have identified solitary hepatic nodules in two surgical specimens and two necropsies. Their sizes ranged from 5-15 $\mathrm{mm}$ in diameter. They were all subcapsular in position on the anterior surface of the liver.

\section{Case reports}

\section{CASE 1}

A man aged 69 yr presented with left sided cerebral infarction and died ten months later of bronchopneumonia. At necropsy, he was found to have adenocarcinoma of the prostate gland with metastases in the regional lymph nodes and the lumbar vertebral column. A single subcapsular nodule was identified on the anterior surface of the left lobe of the liver. It was white on section, measuring $15 \mathrm{~mm}$ in diameter. A thorough search for additional lesions proved fruitless.

\section{CASE 2}

A man aged $45 \mathrm{yr}$ was admitted with left sided hemiplegia due to subarachnoid haemorrhage. After six weeks in hospital, he suddenly deteriorated and died. Necropsy revealed the cause of death to be pulmonary embolism. A single nodule was found on the anterior surface of the right lobe of the liver.

Accepted for publication 14 June 1983
This was white and gritty on section and measured $5 \mathrm{~mm}$ in diameter. No evidence of tumour could be found in any organ.

\section{CASE 3}

A 65-year-old woman, who, two years previously, had had an anterior resection of a rectal carcinoma, presented with local recurrence. At operation a solitary nodule was palpated on the anterior surface of the right lobe of the liver. This was sent for frozen section. Slicing revealed a $12 \mathrm{~mm}$ well circumscribed lemon-yellow nodule with a narrow white rim.

Since this operation eight months ago, the patient has been free of clinical, biochemical and radiological (including CAT scan) evidence of hepatic tumour or local recurrence.

\section{CASE 4}

A 62-year-old man underwent an anterior resection for a Dukes' stage B adenocarcinoma of the colon. During the operation a white 6 millimetre nodule was identified on the anterior aspect of the right lobe of the liver. This was the sole abnormality identifiable in the liver at operation. The patient died of suspected pulmonary embolism five days after the operation and necropsy permission was refused.

\section{Material and methods}

Each specimen was step sectioned and stained with haematoxylin and eosin. Reticulin and elastic preparations were subsequently performed. ZiehlNeelsen, Gram and PAS stains were performed to exclude bacterial infection, including tuberculosis, and fungal infection. 


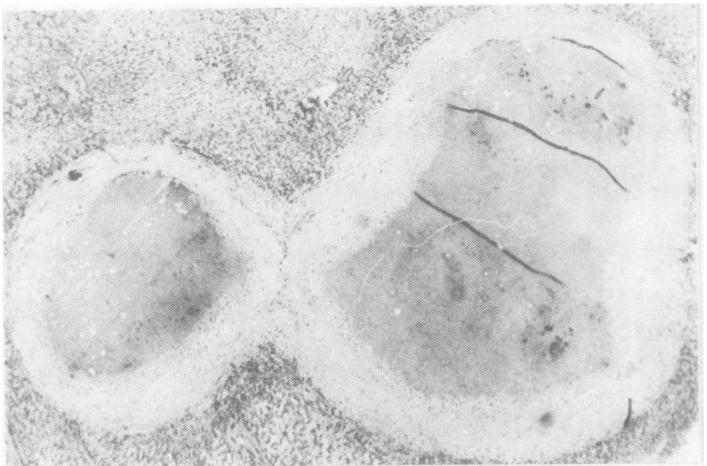

Fig. 1 Low power view of the nodule from case 2 . Note the flecks of calcification within the necrotic core. Haematoxylin and eosin $\times 75$.

\section{MICROSCOPY}

All four speciments shared the histological features of (i) a completely necrotic core (Fig. 1); (ii) a dense hyalinised fibrous capsule (Figs. 1 \& 2); (iii) elastic fibres within the capsule. In addition two of the specimens-cases 2 and 4-showed some calcification of the necrotic core. Two of the lesions-cases 1 and 3-showed abundant reticulin within the core of similar pattern to that of the surrounding liver (Fig. 2). Cases 2 and 4 showed little core reticulin (Fig. 3). These two nodules had much smaller necrotic centres.

The necrotic areas described were eosinophilic and granular and underlying tissue structure was not discernible. Ziehl-Neelsen, Gram and PAS stains failed to reveal bacteria or fungi. There was no evidence of protozoal or helminthic pathogens. No evidence of thrombosis or arteritis was seen in the surrounding liver or elsewhere in any of the cases.

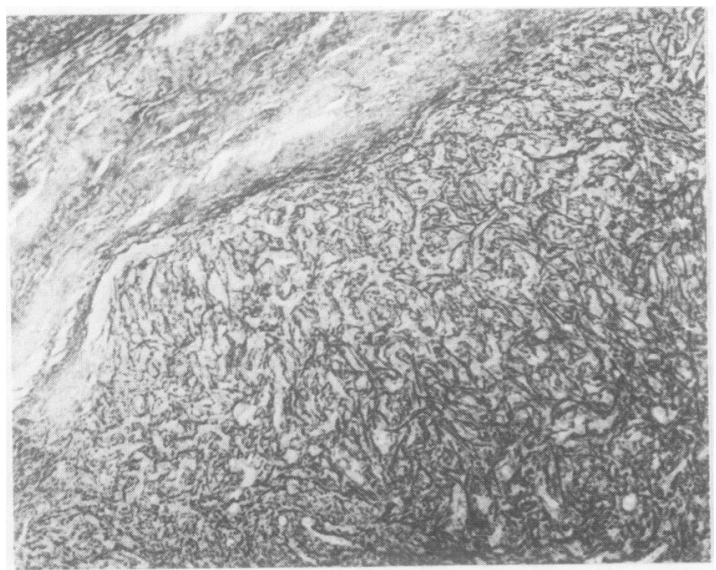

Fig. 2 Reticulin pattern within the necrotic centre of case 1 . Reticulin $\times 75$.

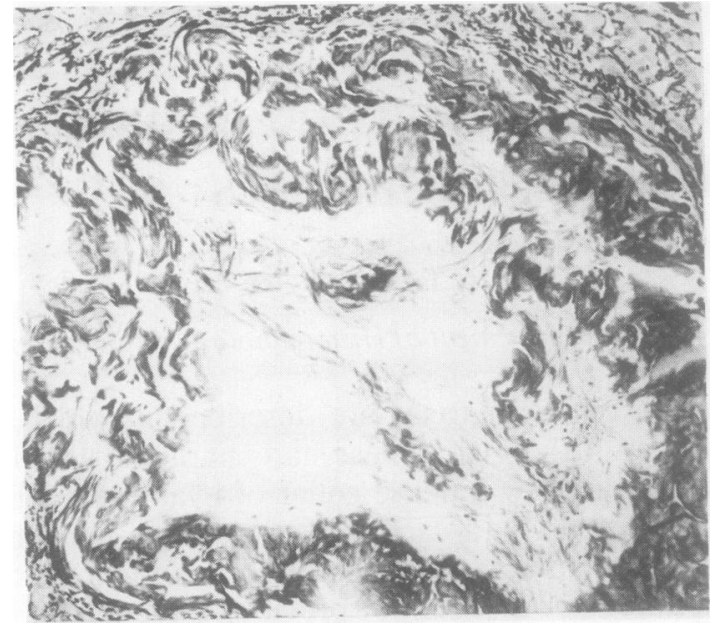

Fig. 3 Paucity of reticulin in the centre of lesion from case 4. Haematoxylin and eosin $\times 120$.

\section{Discussion}

The possibility that these nodules could represent single necrotic metastases must be considered. However the evidence weighs heavily against this. In case 2 there was no evidence of any malignancy. In the other case that came to necropsy-case 1-nos other hepatic lesions were identified. In case 4 the evidence that this lesion was solitary is less compelling. In case 3 recent CT scan (eight months postoperatively) and biochemical tests have not revealed evidence of hepatic metastases or indeed any local recurrence. Also in the experience of this department, a similar lesion has not been seen in the presence of hepatic metastases.

We suggest that two of these lesions have come to light because of the careful palpation of the liver to detect metastases prior to proceeding with colonic resection. It is unlikely that the liver will be examined with such diligence during abdominal operations for benign conditions.

The presence of mature collagen and plentiful elastic fibres in the capsules of these lesions supports our contention that they are of long standing. None of the patients had any evidence of tuberculosis or syphilis, two infections that might be considered as possible causes of these lesions. Indeed two (cases 1 and 3) were known to be WR negative and ZiehlNeelsen stains were negative.

In the belief that such lesions must have been seen before, we have looked at the many surgical and post-mortem specimens in the Museum and we have found one morphologically similar subcapsular lesion measuring $2.5 \mathrm{~cm}$ in diameter. This was a post-mortem case of a 69 -year-old man who died 
from haemoptysis due to carcinoma of the bronchus. There were no metastases in the liver. In 1919 he had contracted amoebiasis while in Mesopotamia and a liver abscess had been drained at this time.

The histological appearances are identical to those of our four cases. No amoebae were detected. To our knowledge none of our four cases has had amoebiasis (and certainly no previous hepatic surgery) or have lived abroad.

We do not know the aetiology of these lesions. Their constant position on the anterior surface of the liver raises the possibility that trauma may be involved. Although one similar lesion has been caused by previous amoebic abscess, it seems unlikely that all the cases are due to "burnt out" amoebiasis and other infective agents may be involved.

Drury' has described fibrous nodules of the liver due to visceral larva migrans (Toxocara and Linguatula spp). These were also found in the spleen and pancreas. Many of the lesions he described were cystic but ours have not been. We have not identified parasitic fragments and the presence of a reticulin pattern in two of our lesions is strongly against a diagnosis of a parasitic aetiology in these instances, at least. Also these two nodules (measuring $12 \mathrm{~mm}$ and $15 \mathrm{~mm}$ in diameter) were larger than those described by Drury.

Other possible causes would include sclerosing haemangiomata, organised thrombi and escaped vegetable material.

\section{Conclusion}

Solitary necrotic nodules must be added to the list of benign lesions, such as cavernous haemangioma, von Meyenburg complexes, solitary adenoma and simple cyst of the liver which can be misinterpreted as hepatic metastases at operation. Some are undoubtedly of parasitic origin but others may be a reaction to trauma or previous chronic infection.

\section{Addendum}

Since submitting this article, we have seen one further case of solitary necrotic nodule of the liver. A 69-year-old man underwent anterior resection for a Dukes' B carcinoma of the rectum and a $0.5 \mathrm{~cm}$ nodule was noted on the anterior surface of the right hepatic lobe. At the time of operation, this was thought to be a haemangioma.

The appearances of the lesion are identical to those we have described. Special stains reveal similar characteristics.

\section{Reference \\ ' Drury RAB. Larval granulomata in the liver. Gut 1962;3:289 94.}

Requests for reprints to: Dr G Lee, Department of Histopathology, St Bartholomew's Hospital, London EC1A 7BE, England. 